\title{
Role of BMI in the Association of the TCF7L2 rs7903146 Variant with Coronary Heart Disease: The Atherosclerosis Risk in Communities (ARIC) Study
}

\author{
Anna M. Kucharska-Newton, ${ }^{1}$ Keri L. Monda, ${ }^{1}$ Suzette J. Bielinski, ${ }^{2}$ \\ Eric Boerwinkle, ${ }^{3}$ Thomas D. Rea, ${ }^{4}$ Wayne D. Rosamond, ${ }^{1}$ James S. Pankow, ${ }^{5}$ \\ Anna Köttgen, ${ }^{6}$ Gerardo Heiss, ${ }^{1}$ and Kari E. North ${ }^{1}$ \\ ${ }^{1}$ Department of Epidemiology, Gillings School of Global Public Health, University of North Carolina at Chapel Hill, \\ Chapel Hill, NC 27514, USA \\ ${ }^{2}$ Division of Epidemiology, Department of Health Services Research, Mayo Clinic, Rochester, MN 55905, USA \\ ${ }^{3}$ Human Genetics Center and Division of Epidemiology, University of Texas, Houston, TX 77225, USA \\ ${ }^{4}$ Department of Medicine, University of Washington, Seattle, WA 98195, USA \\ ${ }^{5}$ Division of Epidemiology and Community Health, University of Minnesota, Minneapolis, MN 55454, USA \\ ${ }^{6}$ Department of Epidemiology, Johns Hopkins University, Baltimore, MD 21205, USA
}

Correspondence should be addressed to Anna M. Kucharska-Newton, anna_newton@unc.edu

Received 29 June 2009; Revised 10 December 2009; Accepted 14 January 2010

Academic Editor: Gianluca Iacobellis

Copyright (C) 2010 Anna M. Kucharska-Newton et al. This is an open access article distributed under the Creative Commons Attribution License, which permits unrestricted use, distribution, and reproduction in any medium, provided the original work is properly cited.

\begin{abstract}
We examined the association of variation in the type 2 diabetes risk-conferring TCF7L2 gene with the risk of incident coronary heart disease (CHD) among the lean, overweight, and obese members of the Atherosclerosis Risk in Communities (ARIC) Study cohort. Cox proportional hazard regression analyses were performed using a general model, with the major homozygote as the reference category. For 9,865 whites, a significant increase in the risk of CHD was seen only among lean $\left(\mathrm{BMI}<25 \mathrm{~kg} / \mathrm{m}^{2}\right)$ individuals homozygous for the $T$ allele of the TCF7L2 rs7903146 gene risk variant (hazard ratio 1.42; 95\% CI 1.03,1.97; $P=.01$ ). No association was found among 3,631 blacks, regardless of BMI status. An attenuated hazard ratio was observed among the nondiabetic ARIC cohort members. This study suggests that body mass modifies the association of the TCF7L2 rs7903146 T allele with CHD risk.
\end{abstract}

\section{Introduction}

Several studies have observed an association between the transcription factor 7-like 2 (TCF7L2) gene and the risk of diabetes [1]. In previous work, using data from the Atherosclerosis Risk in Communities (ARIC) cohort, we reported that the association between the TCF7L2 rs7903146 risk allele $(\mathrm{T})$ and incidence of type 2 diabetes depended on obesity status [2]. Those results were in agreement with data from other studies which suggested a modulating effect of obesity status on the type 2 diabetes susceptibility in TCF7L2 rs7903146 risk allele carriers [3-5]. The modulating effect of obesity status on the association of genetic variants with the risk of type 2 diabetes may in part be related to functional characteristics. Gene variants which affect beta cell function and consequently insulin secretion have a more pronounced effect on the risk of type 2 diabetes among lean and normal weight individuals, whereas gene variants affecting insulin activity at the peripheral cell level increase type 2 diabetes susceptibility among the obese [4].

Individuals with diabetes are at increased risk of cardiovascular morbidity and mortality [6] but the relationship between gene variants conferring type 2 diabetes susceptibility and cardiovascular outcomes has not been sufficiently 
investigated. In this study, we examined whether obesity affects the association of the TCF7L2 rs7903146 gene variant with the risk of incident coronary heart disease (CHD).

\section{Methods and Procedures}

The ARIC Study is a prospective cohort of 15,792 men and women, aged 45-64 years at baseline (1987-1989), selected as a probability sample from four US communities located in North Carolina, Mississippi, Minnesota, and Maryland [7] and followed through ongoing event surveillance, annual follow-up interviews, and three triennial return examinations.

In the present study, we excluded cohort participants who reported race other than white or black $(n=48)$, blacks from the Minnesota and Maryland centers $(n=55)$, those missing TCF7L2 rs7903146 genotype information $(n=$ $1050)$, those with prevalent CHD at baseline $(n=766)$, those missing baseline information on CHD $(n=344)$, and those with body mass index $(\mathrm{BMI})<18.5(n=144)$. The final number of cohort participants after exclusions was 13,496 (9,865 whites and 3,631 blacks), with average follow-up time of $14.9( \pm 3.6)$ years.

Incident $\mathrm{CHD}$, defined as any definite or probable hospitalized myocardial infarction (MI), definite fatal MI, definite or possible fatal CHD, electrocardiographic (ECG) MI (hospitalized occurrence), or coronary revascularization, was ascertained through annual follow-up interviews conducted from baseline examination through December 31, 2004 and reviewed by members of the ARIC Morbidity and Mortality Classification Committee, using published criteria [7]. Identified myocardial infarction cases were validated based on symptoms at presentation, peak cardiac enzyme levels, and Minnesota Code values from three ECG measurements. CHD deaths were ascertained based on death certificates, informant interviews, physician questionnaires, coroner reports, or hospital discharge summaries.

Physical examination of the cohort members and their risk factor assessment were conducted during the baseline visit [8]. Baseline diabetes was defined as either a self-reported physician's diagnosis of diabetes, use of hypoglycemic medications, nonfasting serum glucose levels greater than $200 \mathrm{mg} / \mathrm{dL}$, or fasting ( $\geq 8 \mathrm{hr}$ ) serum glucose level equal to or greater than $126 \mathrm{mg} / \mathrm{dL}$. BMI was determined based on height and weight measured at baseline. BMI cutpoints were chosen a priori according to WHO guidelines [9] for normal weight (18.5-24.99), overweight (25-29.99), and obese $(\geq 30)$. Cohort participants with $\mathrm{BMI}<18.5$ were excluded from analyses due to potential cachexia.

The rs7903146 genotype was determined at the ARIC Study Central DNA Laboratory Health Science Center at Houston, University of Texas School of Public Health, using TaqMan assays (Applied Biosystems, Foster City, CA, USA). Five percent of samples were regenotyped for quality control, and 726 ARIC participants were genotyped in duplicate. The percent agreement was $98 \%$, and the simple $\kappa$ coefficient was 0.97 . Departure from Hardy-Weinberg equilibrium was determined using the $\chi^{2}$ goodness-of-fit test. We modeled the association between the SNP and incident CHD using a general model comparing individuals heterozygous (CT) and homozygous (TT) for the minor T allele to individuals homozygous (CC) for the major $\mathrm{C}$ allele. This model imposes no assumptions of linearity on the relationship between the $\mathrm{T}$ allele and $\mathrm{CHD}$ across genotypes. Associations were determined for each race group separately using Cox proportional hazard regression (adjusted for gender and ARIC study center), with age at time of event as the time variable. We examined the effect of BMI status on the association of the TCF7L2 rs7903146 T allele with the risk of CHD using likelihood ratio tests based on nested models with and without the $\mathrm{BMI}^{*}$ risk allele interaction term. In separate analyses, BMI was coded either as a continuous variable, or as a dichotomous variable (cutpoint at $25 \mathrm{~kg} / \mathrm{m}^{2}$ ). We used similar models of likelihood ratio tests to further examine potential effect modification due to high-density lipoprotein (HDL)-cholesterol, diabetes, cigarette smoking, and hypertension.

\section{Results}

The minor allele $(\mathrm{T})$ frequencies were $8.7 \%$ for the total sample population, $8.9 \%$ among whites, and $8.3 \%$ among blacks. The genotype distributions at rs7903146 conformed to Hardy-Weinberg equilibrium in the entire sample and within race strata. Baseline characteristics of the sample, stratified by race, are presented in Table 1 . Mean BMI values differed by genotype in the total sample $(P=.03)$, but not within race strata (whites $P=.11$; blacks $P=.28$ ).

Although we observed no significant association of the rs7903146 TCF7L2 gene variant with CHD overall (Table 2, $P=.22$ ), we observed that BMI, coded as a continuous variable, modified the association of the rs7903146 TCF7L2 gene variant with the relative hazard of CHD among whites $\left(P_{\text {interaction }}<.01\right)$, but not among blacks $\left(P_{\text {interaction }}=.45\right)$. A similar pattern of effect modification was observed with BMI coded as a dichotomous variable using the clinically defined overweight cutpoint $\left(25 \mathrm{~kg} / \mathrm{m}^{2}\right)$.

A statistically significant association of the TCF7L2 rs7903146 gene variant with the relative hazard of CHD was observed only among persons with BMI $<25 \mathrm{~kg} / \mathrm{m}^{2}$ (0020HR 1.40 (95\% CI 1.04, 1.88)) (Table 2). This association was significant among whites (HR $1.42(95 \%$ CI 1.03, 1.97)), but not among blacks (HR 1.26 (95\% CI 0.58, 2.71)). A similar pattern was observed for the association of the rs7903146 risk allele with CHD across gender-specific tertiles of waist circumference and subscapular skinfold thickness.

We did not observe a statistically significant effect of diabetes on the association of the TCF7L2 rs7903146 gene variant with the risk of $\mathrm{CHD}\left(P_{\text {interaction }}=.40\right)$. Limiting analysis to lean and normal weight $\left(\mathrm{BMI}<25 \mathrm{~kg} / \mathrm{m}^{2}\right)$ cohort members who were free of diabetes resulted in a relative hazard of CHD among the carriers of the risk genotype of $1.33(95 \%$ CI $0.94,1.88)$ for the total sample and $1.36(95 \%$ CI $0.94,1.96)$ among whites. When assuming the recessive mode of inheritance (CC and CT alleles as the referent), we found that the hazard ratio of CHD among lean and normal 
TABLE 1: Baseline characteristics of the sample population (whites and blacks combined) according to TCF7L2 genotype, the ARIC study cohort.

\begin{tabular}{lccc}
\hline Characteristic & CC & CT & TT \\
& $n=6,736$ & $n=5,583$ & $n=1,177$ \\
\hline Age (years) & $54.0(5.7)$ & $53.9(5.8)$ & $54.1(5.8)$ \\
BMI $\left(\mathrm{kg} / \mathrm{m}^{2}\right)$ & $27.8(5.4)$ & $27.6(5.3)$ & $27.5(4.9)$ \\
HDL-cholesterol (mmol/L) & $1.4(0.4)$ & $1.4(0.4)$ & $1.3(0.4)$ \\
Fasting glucose (mmol/L) & $6.0(2.2)$ & $6.1(2.2)$ & $6.2(2.3)$ \\
Gender (\% Male) & 42.8 & 43.9 & 44.8 \\
Race (\% Black) & 26.9 & 27.2 & 25.7 \\
Diabetes (\%) & 9.6 & 34.6 & 14.5 \\
Hypertension (\%) & 33.7 & 57.4 & 31.1 \\
Ever smoker (\%) & 56.5 & 58.6 \\
\hline
\end{tabular}

${ }^{*}$ differences across genotype strata were significant at $\alpha=0.05$ only for BMI $(P=.03)$, diabetes $(P<.01)$, and fasting glucose $(P<.01)$.

TABle 2: Association of the TT and CT genotypes of the TCF7L2 rs7903146 gene variant with the incidence of coronary heart disease in categories of BMI. Cox proportional hazard analyses were performed with age at time of event as the time variable and adjustment for gender and ARIC study center. For the total sample and in race strata, the referent category was individuals with BMI $<25 \mathrm{~kg} / \mathrm{m}^{2}$ carrying the CC genotype of the TCF7L2 rs7903146 gene variant.

\begin{tabular}{|c|c|c|c|c|c|c|c|c|c|}
\hline & \multicolumn{3}{|c|}{ Total sample $(n=13,496)$} & \multicolumn{3}{|c|}{ Whites $(n=9,865)$} & \multicolumn{3}{|c|}{ Blacks $(n=3,631)$} \\
\hline & $\begin{array}{c}\text { CC } \\
(n=6,736)\end{array}$ & $\begin{array}{c}\mathrm{CT} \\
(n=5,583)\end{array}$ & $\begin{array}{c}\mathrm{TT} \\
(n=1,177)\end{array}$ & $\begin{array}{c}\text { CC } \\
(n=4,927)\end{array}$ & $\begin{array}{c}\mathrm{CT} \\
(n=4,063)\end{array}$ & $\begin{array}{c}\mathrm{TT} \\
(n=875)\end{array}$ & $\begin{array}{c}\text { CC } \\
(n=1,809)\end{array}$ & $\begin{array}{c}\mathrm{CT} \\
(n=1,520)\end{array}$ & $\begin{array}{c}\mathrm{TT} \\
(n=302)\end{array}$ \\
\hline Events & 883 & 717 & 172 & 691 & 551 & 129 & 192 & 166 & 43 \\
\hline p-years & 100,435 & 82,899 & 17,384 & 74,421 & 61,053 & 13,112 & 26,015 & 21,846 & 4,272 \\
\hline \multirow{2}{*}{$\mathrm{HR}(95 \% \mathrm{CI})$} & & 1.02 & 1.11 & & 1.00 & 1.07 & & 1.08 & 1.24 \\
\hline & 1.00 & $(0.92,1.12)$ & $(0.94,1.31)$ & 1.00 & $(0.89,1.11)$ & $(0.89,1.30)$ & 1.00 & $(0.88,1.33)$ & $(0.89,1.72)$ \\
\hline \multirow{2}{*}{\multicolumn{10}{|c|}{$\begin{array}{l}\text { BMI categories: } \\
\text { BMI < } 25 \\
(n=4,476)\end{array}$}} \\
\hline & & & & & & & & & \\
\hline Events & 210 & 159 & 55 & 175 & 124 & 47 & 35 & 35 & 8 \\
\hline p-years & 33,083 & 28,2006 & 6,033 & 27,850 & 23,577 & 5,134 & 5,232 & 4,629 & 900 \\
\hline \multirow{2}{*}{$\operatorname{HR}(95 \% \mathrm{CI})$} & & 0.90 & 1.40 & & 0.82 & 1.42 & & 1.31 & 1.26 \\
\hline & 1.00 & $(0.74,1.11)$ & $(1.04,1.88)$ & 1.00 & $(0.65,1.04)$ & $(1.03,1.97)$ & 1.00 & $(0.82,2.09)$ & $(0.58,2.71)$ \\
\hline \multicolumn{10}{|l|}{$\begin{array}{l}\text { BMI 25-30 } \\
(n=5,325)\end{array}$} \\
\hline Events & 394 & 320 & 67 & 324 & 260 & 52 & 70 & 60 & 15 \\
\hline$p$-years & 39,309 & 32,918 & 6,989 & 29,446 & 24,454 & 5,399 & 9,862 & 8,464 & 1,590 \\
\hline $\mathrm{HR}(95 \% \mathrm{CI})$ & 1.33 & 1.31 & 1.28 & 1.31 & 1.28 & 1.18 & 1.22 & 1.25 & 1.55 \\
\hline ח & $(1.12,1.57)$ & $(1.10,1.56)$ & $(0.97,1.69)$ & $(1.09,1.58)$ & $(1.06,1.56)$ & $(0.86,1.61)$ & $(0.81,1.83)$ & $(0.82,1.89)$ & $(0.85,2.85)$ \\
\hline \multicolumn{10}{|l|}{$\begin{array}{l}\mathrm{BMI} \geq 30 \\
(n=3,695)\end{array}$} \\
\hline Events & 279 & 238 & 50 & 192 & 167 & 30 & 87 & 71 & 20 \\
\hline p-years & 28,045 & 21,776 & 4,361 & 17,124 & 13,023 & 2,579 & 10,920 & 8,753 & 1,782 \\
\hline HR $(95 \%$ CI) & 1.61 & 1.85 & 1.89 & 1.56 & 1.95 & 1.71 & 1.57 & 1.53 & 2.02 \\
\hline & $(0.35,1.93)$ & $(1.53,2.23)$ & $(1.39,2.57)$ & $(0.27,1.91)$ & $(1.58,2.41)$ & $(1.16,2.53)$ & $(1.06,2.34)$ & $(1.01,2.30)$ & $(1.16,3.51)$ \\
\hline
\end{tabular}

weight whites with diabetes was 2.13 (95\% CI 1.00, 4.55) as compared to the hazard ratio of CHD among lean and normal weight whites without diabetes (HR 1.35 (95\% CI $0.96,1.91)$ ).

Effect measure modification of the association of the TCF7L2 rs7903146 gene variant with the risk of CHD is stronger for nonfatal MI as compared to total $\mathrm{CHD}$, which in this study included, in addition to myocardial infarction, fatal CHD, silent MI, and coronary procedures. The hazard ratio for the association of this polymorphism with the risk of myocardial infarction among the lean and normal weight ARIC cohort participants was 1.72 (95\% CI 1.14, 2.60). 


\section{Discussion}

Our results suggest that among individuals homozygous for the T allele of the TCF7L2 rs7903146 gene variant, the relative hazard of coronary heart disease may be modestly increased among the lean and normal weight individuals, but not among the overweight or the obese. We observed this association in the total sample and among whites; however, we did not observe any pattern of association among the blacks of this study, possibly due to insufficient statistical power; we calculated that we had only $10 \%$ power to detect a hazard ratio of 1.6 among blacks.

This study expands on that of Bielinski et al. [10], in which the authors examined the association of variants of the TCF7L2 gene with the risk of coronary heart disease and found a significant level of association only among whites with diabetes and only with additional adjustment for BMI. The present finding of a modest association of a variant in the TCF7L2 gene with increased relative hazard of coronary heart disease is consistent with the greater risk of cardiovascular disease in those with, or at risk of diabetes. One can speculate based on this, that a genetic predisposition towards development of diabetes may carry an increased risk of coronary heart disease. It is difficult in population studies to answer the question to what degree TCF7L2 influences cardiovascular risk independently or through diabetes. We attempted to address this question by stratifying our analyses by diabetes status and by increasing the power of analysis with the assumption of recessive mode of inheritance for this gene. However, such adjustment strategies are crude and our sample size was too small to disentangle the possible role of diabetes in the association of TCF7L2 with CHD in this population.

We examined the association of the TCF7L2 gene variant with the risk of CHD across strata of tertiles of waist circumference and subscapular skinfold thickness, which are often used as more direct measures of visceral or subcutaneous adipose tissue. Although the associations were weaker than in strata of BMI, the patterns of association were similar, suggesting that the association is related to overall adiposity rather than more specifically to subcutaneous or visceral fat. If replicated in other studies, this observation would be consistent with results obtained from studies of the association of genetic variation in the TCF7L2 gene and diabetes [4]. The TCF7L2 gene is part of the Wnt signaling pathway [11] a critical pathway in cell development which regulates, among others, the activity of glucagonlike peptide-1 (GLP-1) [12]. Presence of genetic variants in the TCF7L2 gene is associated with decreased insulin levels [13], an effect that may be a consequence of a decrease in stimulation of insulin release by GLP-1. This is a direct effect on beta cell function that is independent of obesityinduced insulin resistance and subsequent impaired insulin secretion and gluconeogenesis. Impaired insulin secretion and abnormal plasma glucose levels, observed in diabetes, represent dysregulation of multiple metabolic pathways. It is thus possible that the mechanism of diabetes is different in lean as compared to obese individuals, in whom inputs from other metabolic dysfunctions contribute additional impairment. The rs7903146 SNP is not in the coding region of the TCF7L2 gene therefore the modality by which it imparts increased susceptibility to type 2 diabetes is difficult to attribute. It is equally difficult to identify the specific function of obesity status in the association of the TCF7L2 gene with incidence of diabetes. The TCF7L2 factor can affect expression of the Wnt signaling pathway thus leading to increased adipogenesis [14] and in that way may contribute to a milieu conducive to the development of diabetes.

The above mentioned mechanisms, suggestive of a pleiotropic effect of the TCF7L2 gene, may differentially contribute to increased risk of coronary heart disease. Replication of our results in other, preferably larger, cohorts would broaden the understanding of the increased risk of cardiovascular disease in individuals with diabetes and those at increased risk of diabetes.

\section{Abbreviations}
ARIC: Atherosclerosis Risk in Communities
CHD: coronary Heart Disease
SNP: single nucleotide polymorphism
BMI: body mass index.

\section{Acknowledgments}

The Atherosclerosis Risk in Communities Study is carried out as a collaborative study supported by National Heart, Lung, and Blood Institute contracts N01-HC-55015, N01-HC-55016, N01-HC-55018, N01-HC-55019, N01-HC55020, N01-HC-55021, and N01-HC-55022. The authors thank the staff and participants of the ARIC study for their important contributions. This work was supported by a National Service Award Training Grant T32-H2-007055.

\section{References}

[1] S. Cauchi and P. Froguel, "TCF7L2 genetic defect and type 2 diabetes," Current Diabetes Reports, vol. 8, no. 2, pp. 149-155, 2008.

[2] Y. Yan, K. E. North, C. M. Ballantyne, et al., "Transcription factor 7-like 2 (TCF7L2) polymorphism and context-specific risk of type 2 diabetes in African American and Caucasian adults: the atherosclerosis risk in communities (ARIC) study," Diabetes, vol. 58, pp. 285-289, 2008.

[3] S. Cauchi, H. Choquet, R. Gutierrez-Aguilar, et al., "Effects of TCF7L2 polymorphisms on obesity in European populations," Obesity, vol. 16, no. 2, pp. 476-482, 2008.

[4] S. Cauchi, K. T. Nead, H. Choquet, et al., "The genetic susceptibility to type 2 diabetes may be modulated by obesity status: implications for association studies," BMC Medical Genetics, vol. 9, article 45, 2008.

[5] A. Helgason, S. Palsson, G. Thorleifsson, et al., "Refining the impact of TCF7L2 gene variants on type 2 diabetes and adaptive evolution," Nature Genetics, vol. 39, no. 2, pp. 218225, 2007.

[6] J. Stamler, O. Vaccaro, J. D. Neaton, and D. Wentworth, "Diabetes, other risk factors, and 12-yr cardiovascular mortality for men screened in the multiple risk factor intervention trial," Diabetes Care, vol. 16, no. 2, pp. 434-444, 1993. 
[7] The ARIC Investigators, "The Atherosclerosis Risk in Community (ARIC) study. Design and objectives," American Journal of Epidemiology, vol. 129, pp. 687-702, 1989.

[8] Atherosclerosis Risk in Communities Study Manual E.

[9] "Obesity: preventing and managing the global epidemic," Report of a WHO Consultation on Obesity, World Health Organization, Geneva, Switzerland, 1998, Consulation on Obesity.

[10] S. J. Bielinski, J. S. Pankow, A. R. Folsom, K. E. North, and E. Boerwinkle, "TCF7L2 single nucleotide polymorphisms, cardiovascular disease and all-cause mortality: the Atherosclerosis Risk in Communities (ARIC) study," Diabetologia, vol. 51, no. 6, pp. 968-970, 2008.

[11] F. Yi, J. Sun, G. E. Lim, I. G. Fantus, P. L. Brubaker, and T. Jin, "Cross talk between the insulin and Wnt signaling pathways: evidence from intestinal endocrine L cells," Endocrinology, vol. 149, no. 5, pp. 2341-2351, 2008.

[12] S. A. Schafer, O. Tschritter, F. Machicao, et al., "Impaired glucagon-like peptide-1-induced insulin secretion in carriers of transcription factor 7-like 2 (TCF7L2) gene polymorphisms," Diabetologia, vol. 50, no. 12, pp. 2443-2450, 2007.

[13] M. K. Loder, G. D. S. Xavier, A. McDonald, and G. A. Rutter, "TCF7L2 controls insulin gene expression and insulin secretion in mature pancreatic $\hat{\mathrm{I}}^{2}$-cells," Biochemical Society Transactions, vol. 36, no. 3, pp. 357-359, 2008.

[14] S. E. Ross, N. Hemati, K. A. Longo, et al., "Inhibition of adipogenesis by Wnt signaling," Science, vol. 289, no. 5481, pp. 950-953, 2000. 


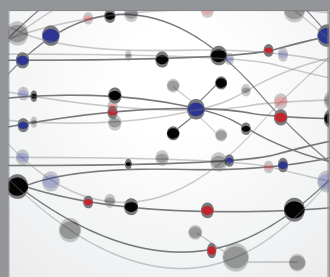

The Scientific World Journal
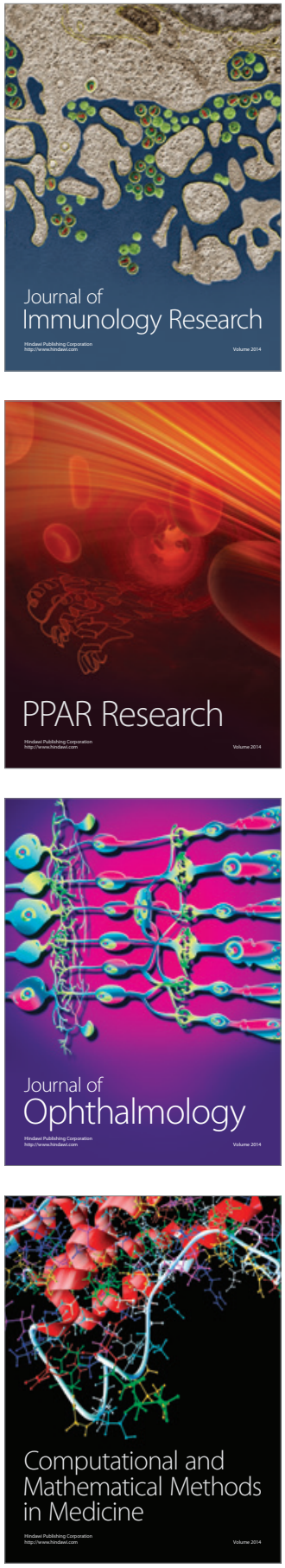

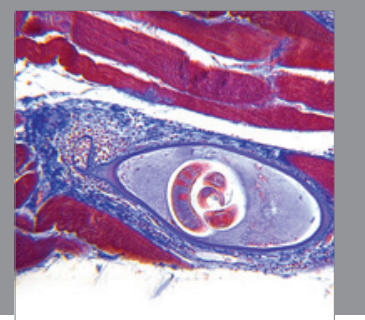

Gastroenterology

Research and Practice
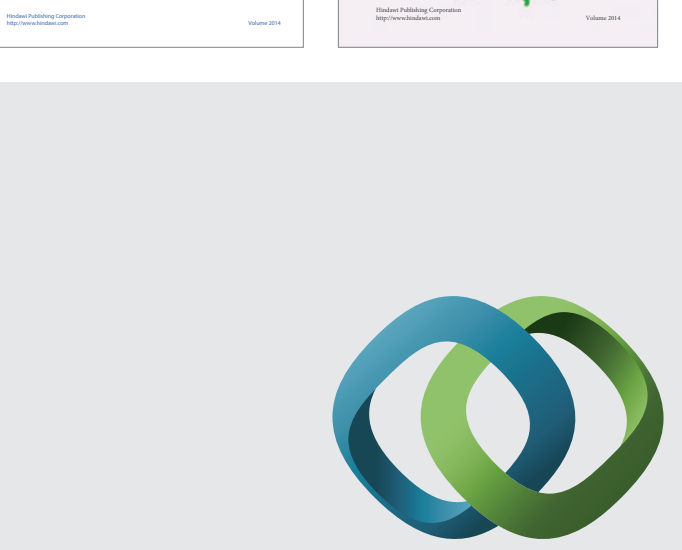

\section{Hindawi}

Submit your manuscripts at

http://www.hindawi.com
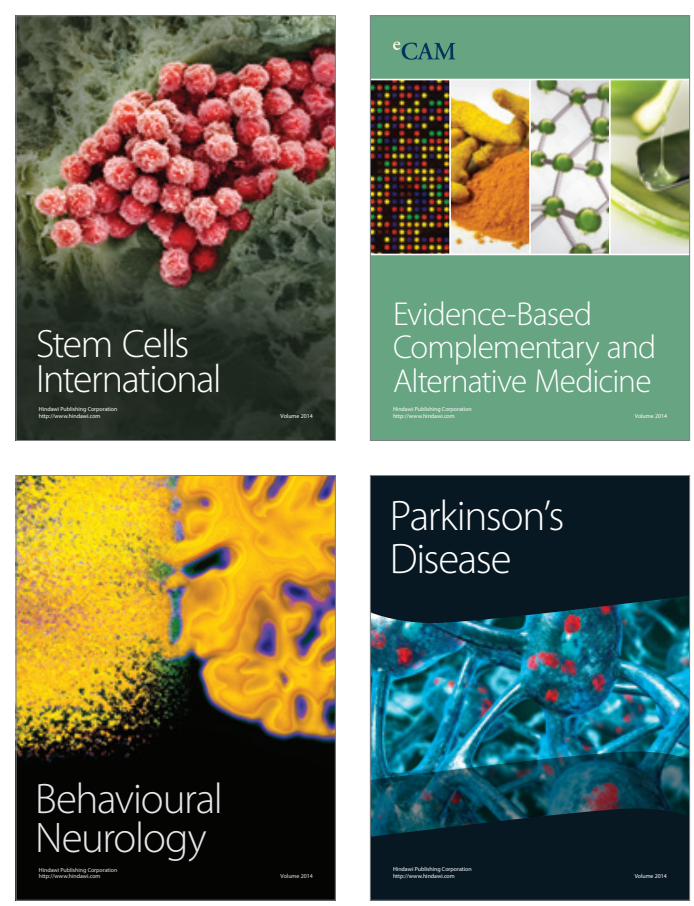

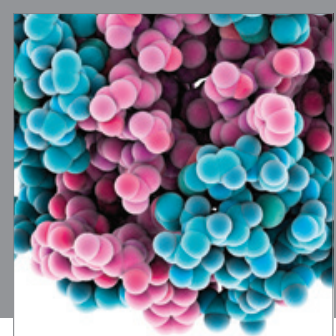

Journal of
Diabetes Research



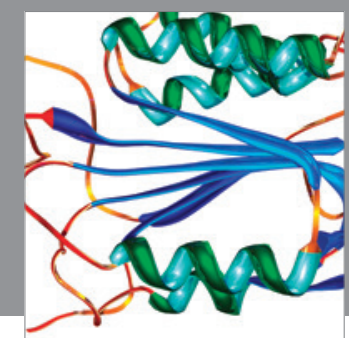

Disease Markers
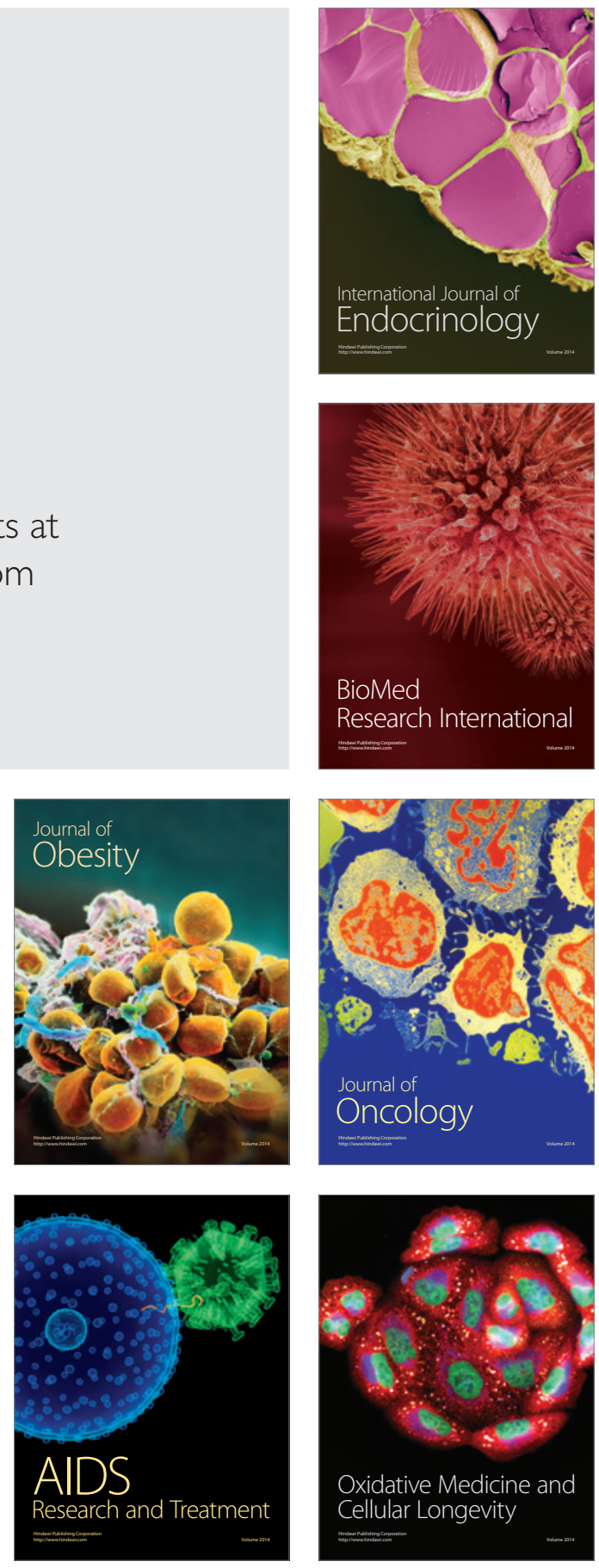